\title{
Análisis multifactorial de los factores de riesgo de bajo peso al nacer en Salvador, Bahia
}

\author{
Jorge José Santos Pereira Solla, ${ }^{1}$ Rosana Aquino Guimarães Pereira, ${ }^{1}$ \\ María Guadalupe Medina, ${ }^{1}$ Lorene Louise S. Pinto ${ }^{1}$ \\ y Eduardo Mota $^{1}$
}

RESUMEN El presente estudio constituye un análisis multifactorial de los factores de riesgo de bajo peso al nacer en un grupo de recién nacidos en una zona urbana del Brasil. Se incluyeron en el estudio un total de 1023 nacidos vivos, dados a luz en cuatro maternidades de Salvador, Bahia, entre julio de 1987 y febrero de 1988. Las fuentes de información fueron las historias clinicas y las entrevistas con las madres en la maternidad. El análisis se realizó mediante regresión logística. En el modelo final los factores de riesgo incluidos fueron los siguientes: edad materna menos de 21 años o más de 35; edad gestacional menos de 38 semanas; resultado desfavorable del embarazo anterior; intervalo intergenésico previo de 12 meses o menos; tabaquismo; e hipertensión. Se presentan los valores del riesgo atribuible poblacional para los factores de riesgo incluidos en el modelo final. Esos factores deben emplearse para detectar a las gestantes con alto riesgo de dar a luz un niño de bajo peso, a las que debe brindarse mayor atención prenatal.

Varios estudios han mostrado la asociación del bajo peso al nacer (BPN) o insuficiencia ponderal neonatal con la mayor frecuencia durante el primer año de vida de diversos trastornos, entre los que destacan el retraso del desarrollo neurológico, la hemorragia cerebral, las alteraciones respiratorias y otras enfermedades que provocan la necesidad de atención prolongada y hospitalización (1-4). También hay muchos datos que prueban la asociación del bajo peso al nacer con una

\footnotetext{
1 Instituto de Salud Colectiva, Universidad Federal de Bahia, Brasil. La correspondencia relacionada con este artículo debe dirigirse al primer autor a la siguiente dirección postal: Instituto de Saúde Coletiva, Universidade Federal da Bahia, Rua Padre Feijó, 29, 4o. andar, Canela-40110, Salvador, Bahia, Brasil.
}

mayor mortalidad infantil (4-8). Estudios epidemiológicos más recientes muestran una posible asociación entre el BPN y enfermedades del adulto tales como la hipertensión arterial y la diabetes mellitus no insulinodependiente (9).

Además, la frecuencia con la que ocurre el BPN puede considerarse un indicador general del estado de salud de una población, ya que dicha frecuencia es sensible a las condiciones socioeconómicas adversas. Por ejemplo, en la India se estima una incidencia de 30\% de BPN (10). En 1982 la OMS estimó una incidencia de BPN de 9\% en el Brasil (11). En Araquara (municipio del estado de São Paulo) se ha estimado una frecuencia de BPN de 9,2\% en 1986 (12). En el estado de Rio Grande do Sul se estimó un 9,6\% de
BPN en 1991 y un 8,2\% en 1992 (13). En un conjunto de cinco municipios del estado de São Paulo se estimó una frecuencia de 8,5\% de BPN en 1992 (14). En la Investigación Nacional sobre Salud y Nutrición se estimó un 10\% de BPN en 1989 en todo el Brasil (15).

Se han estudiado numerosos factores de riesgo de $\mathrm{BPN}$ : madre de menos de 20 años (16-18) o de más de 40 (18), desnutrición materna (19-22), infección durante la gestación (23-25), neonatos de bajo peso $u$ otros resultados desfavorables en gestaciones anteriores $(18,26-28)$, intervalo intergenésico corto (18), parto prematuro (18) y consumo de tabaco durante la gestación (16-19, 26, 28-32).

Los modelos más completos de determinación del BPN fueron los utilizados por Barros et al. (26), Benício et 
al. (17), Lee et al. (33) y Stüsser et al. (34). Barros et al. determinaron mediante la técnica de regresión que la variable más importante asociada con el BPN era el peso materno al final de la gestación, siguiéndole en orden de importancia la edad gestacional, el tabaquismo durante el embarazo, sexo del bebé, antecedentes de haber tenido otro hijo de bajo peso y el peso materno al inicio de la gestación (26). Benício et al. realizaron un análisis multifactorial y hallaron que, una vez controladas las demás variables, los factores que presentaron asociación con BPN fueron la ausencia de atención prenatal, el tabaquismo durante el embarazo, el peso materno pregestacional de menos de $50 \mathrm{~kg}$, la edad materna de menos de 20 años y la baja escolaridad materna asociada a carencia de atención prenatal (17). Lee et al. determinaron mediante regresión logística la asociación estadísticamente significativa de cinco factores (raza, estado civil, escolaridad, número previo de partos y atención prenatal) con el BPN (33). Stüsser et al. llevaron a cabo un estudio de casos y controles en La Habana, Cuba, en el que mediante análisis de la varianza, regresión lineal múltiple y regresión logística investigaron el riesgo relativo correspondiente a 107 factores de riesgo hipotéticos de BPN (34).

En el presente estudio se examinaron los factores de riesgo en conjunto, identificando los que contribuyen más a incrementar el riesgo en un grupo de niños nacidos en una zona urbana.

\section{METODOLOGÍA}

Se estudiaron 1023 nacidos vivos en Salvador, Bahia, entre julio de 1987 y febrero de 1988, en cuatro maternidades (tres públicas y una privada) de esa ciudad. Se recopiló información sobre el estado del neonato y las variables relacionadas con las condiciones maternas durante la gestación. Dos de las maternidades son parte de la red de servicios sanitarios de la Secretaría de Salud del Estado de Bahía y atienden fundamentalmente a la población de bajos ingresos que reside en barrios marginales de condiciones precarias en cuanto a vivienda y saneamiento. La otra maternidad pública, integrada en el sistema de Previsión Social en la época del estudio, se halla en un barrio central de la ciudad y atiende a una población en la que predominan los asalariados urbanos, cuyas condiciones de vida son mejores que las de la población atendida en las otras dos maternidades citadas. La maternidad privada pertenece a un seguro médico privado y atiende a personas cuyos ingresos son en general mayores que los de las personas atendidas en otras maternidades. Estas instituciones se escogieron para ofrecer un perfil variado de las características sociodemográficas de la población, ya que en Salvador más de $90 \%$ de los partos son institucionales y esas maternidades agrupan aproximadamente un 12\% del total anual de partos (35). Según datos de la Secretaría de Salud del Estado de Bahía (no publicados), en 1987 se registraron 40590 admisiones en 11 de las 15 instituciones hospitalarias que dan asistencia al parto en Salvador. Ese mismo año se estima que hubo un total de 59334 nacidos vivos en la ciudad.

Las fuentes de información utilizadas fueron las historias clínicas y entrevistas con las embarazadas en la maternidad, con consentimiento de la administración de cada una de las maternidades. Para la selección de la muestra se usó el registro de parturientas admitidas en las 24 horas anteriores al inicio de la recogida de datos cada día que se visitó la maternidad, incluyéndose en la muestra los 12 primeros partos de ese período. Se excluyeron los mortinatos y las madres que no residían en la ciudad. Se procedió a aplicar el primer cuestionario en la enfermería o en la sala de la maternidad, una vez obtenido el consentimiento materno. Esta fase se llevó a cabo entre julio de 1987 y febrero de 1988. Se registró un total de 1023 parejas de madre y neonato, 655 (64\%) procedentes de las maternidades de la Secretaría de Salud del Estado, 221 (22\%) de la maternidad de la Previsión
Social y las restantes 147 (14\%) de la maternidad privada. A cada mes de trabajo en las maternidades correspondió un grupo de seguimiento. El primer grupo se visitó en su domicilio por primera vez en noviembre de 1987. La fase de visitas se cerró en febrero de 1988.

En el primer protocolo que se utilizó se incluyeron datos de identificación completa de la familia - con dirección detallada y referencias que permitieran la localización posterior del domicilio-, los antecedentes obstétricos y patológicos previos a la gestación actual y durante la misma, las características de la gestación, parto y puerperio, la utilización de los servicios de atención prenatal y las características del neonato en su primer día de vida. Se visitó a cada participante a intervalos de cuatro meses. Los cuestionarios rellenados fueron revisados y los datos faltantes se completaron en visitas posteriores. El equipo de recolección de datos estuvo formado por estudiantes de medicina y enfermería. Una vez seleccionados los entrevistadores, se les dio formación sobre cómo aplicar los cuestionarios y cómo estandarizar los procedimientos de recogida de datos. Los protocolos de registro y de visitas fueron archivados por número de identificación del neonato. Un mapa de visitas permitió mantener actualizadas las labores de recogida de datos y control de casos de participación rehusada y de direcciones no localizadas.

El peso neonatal se obtuvo de la historia clínica. Se definió el BPN como un peso igual a $2500 \mathrm{~g}$ o menor, independientemente de la edad gestacional. A partir de 1976 la OMS modificó la definición de BPN a un peso al nacer "menor que $2500 \mathrm{~g}$ (hasta $2499 \mathrm{~g}$ inclusive)" (36). En este estudio se optó por la mencionada definición debido a la tendencia a registrar los pesos observados aproximando al número entero inmediato en decigramos o hectogramos. Consideramos que la opción de usar un peso menor o igual a $2500 \mathrm{~g}$ era una forma de evitar la subestimación del BPN, en la medida que trabajábamos con datos 
recogidos a partir de historias clínicas de maternidades.

Se investigaron las siguientes variables que se consideraron a priori posibles factores de riesgo de BPN:

- intervalo desde la anterior gestación (intervalo intergenésico) de 12 meses o menos;

- anterior gestación con resultado desfavorable;

- edad gestacional de menos de 38 semanas;

- madre de menos de 21 años o más de 35;

- tabaquismo durante el embarazo;

- menos de seis consultas de atención prenatal;

- hipertensión durante el embarazo;

- madre de estatura baja ( $\leq 1,5 \mathrm{~m})$;

- más de tres embarazos previos.

Cada madre que presentó una de estas circunstancias se consideró "expuesta" al factor correspondiente.

Se consideró que el resultado de la anterior gestación había sido desfavorable si se produjo un aborto, un nacido vivo prematuro o un mortinato. Para delimitar estas tres categorías diagnósticas se usaron las definiciones de la novena revisión de la Clasificación Internacional de Enfermedades (37).

Se calculó inicialmente el riesgo relativo (RR) no ajustado de $\mathrm{BPN}$ correspondiente a cada factor individual, dividiendo la frecuencia de BPN en los casos expuestos por la frecuencia en los no expuestos.

A continuación se calculó mediante regresión logística la razón de posibilidades $(\psi)$ no ajustada correspondiente a cada factor individual. ${ }^{2}$ Por último, se realizó el análisis multifactorial mediante regresión logística en la que

\footnotetext{
2 Se usará aquí "razón de posibilidades" para lo que en inglés se denomina odds ratio, que se abreviará $\psi$ (psi). Esa letra griega ha sido utilizada como símbolo de este concepto matemático por autores como Schlesselman (Case-control studies: design, conduct, analysis, Nueva York, Oxford University Press, 1982), Armitage y Berry (Statistical methods in medical research, Oxford, Blackwell, 1987) y Breslow y Day (Statistical methods in cancer research, Vol. I-The analysis of case-control studies, Lyon, IARC, 1980) (N. del editor).
}

se incluyeron todos los factores que habían mostrado asociación con el $\mathrm{BPN}$ en los análisis individuales. Se utilizó el procedimiento de adición de variables una a una (procedimiento denominado en inglés forward stepwise regression). En cada paso se utilizó un criterio de probabilidad de 0,05 para añadir una variable y de 0,10 para eliminarla.

La regresión logística es el modelo matemático más utilizado cuando la medida del efecto es una variable dicotómica y se intenta establecer una relación entre una o más variables de exposición y una enfermedad. La regresión logística multifactorial permite estudiar el efecto conjunto de la exposición a distintos factores, controlando a la vez el fenómeno de confusión. La razón de posibilidades $(\psi)$ es la única medida de asociación directamente estimada en un modelo logístico y no requiere condiciones especiales. La $\psi$ que estima el riesgo de BPN para cada factor se derivó a partir de los coeficientes de la regresión logística (38-39). El análisis se llevó a cabo con el programa SPSS.

Para estimar el efecto de la reducción de incidencia de BPN que podría lograrse eliminando la exposición de toda la población al factor de riesgo se estimó el riesgo atribuible poblacional (RAP) porcentual según la fórmula de Levin (40-41). Tal cálculo se realiza partiendo del valor de $\psi$ estimado y de la proporción de la población expuesta al factor de riesgo. El riesgo atribuible es la proporción máxima de incidencia de la enfermedad que puede ser atribuida a un factor etiológico (41).

\section{RESULTADOS}

El peso al nacer en la muestra estudiada tuvo una media de $3138 \mathrm{~g}$, una mediana de $3150 \mathrm{~g}$ y una desviación estándar de 505 g. Se halló BPN en $8,9 \%$ de los neonatos.

La brevedad del período de gestación (<38 semanas) o del intervalo intergenésico $(<13$ meses desde el último embarazo), el resultado desfavorable de la anterior gestación, el taba- quismo durante el embarazo, la gestante muy joven ( $\leq 20$ años) o de edad avanzada ( $\geq 36$ años), o de estatura baja $(\leq 1,5 \mathrm{~m})$, o hipertensa, y la atención prenatal deficiente ( $<6$ consultas) fueron factores asociados al BPN, con RR > 1,0. No se halló asociación entre el BPN y el antecedente de más de tres gestaciones previas (cuadro 1).

En modelos de regresión logística con una sola variable explicativa el valor de $\psi$ no ajustado estimado para la asociación entre los factores de riesgo estudiados y el BPN fue muy similar al RR no ajustado. En ambos análisis las asociaciones positivas (es decir, RR $>1,0$ o bien $\psi>1,0)$ fueron estadísticamente significativas con todos los factores, excepto la estatura materna baja, la hipertensión durante el embarazo y una atención prenatal de menos de seis consultas (cuadros 1 y 2).

En el modelo multifactorial de regresión logística se incluyeron todas las variables analizadas excepto la variable "más de tres gestaciones previas", que no había mostrado asociación con el BPN en los análisis individuales.

En el modelo multifactorial final (cuadro 3) todas las estimaciones de $\psi$ excedieron de la unidad y la asociación fue estadísticamente significativa para todos los factores, incluso la hipertensión durante el embarazo y la estatura materna baja, que habían mostrado una asociación positiva no estadísticamente significativa en el análisis individual.

El modelo indica que nacer con una edad gestacional de menos de 38 semanas, o después de un intervalo de 12 meses o menos desde la anterior gestación, o de una madre cuyo anterior embarazo tuvo un resultado desfavorable, o que presentó hipertensión durante el embarazo, eleva a más del doble la probabilidad de BPN.

El riesgo atribuible poblacional (cuadro 3, última columna) está calculado bajo el supuesto de que la proporción de expuestos en la muestra es representativa de la proporción de expuestos en la población. El tamaño muestral apunta a favor de este supuesto, ya que la muestra es grande en 
CUADRO 1. Proporción de expuestos y no expuestos con bajo peso al nacer (BPN) en 1023 nacidos vivos en Salvador, Bahia, 1987-1988, con los correspondientes riesgos relativos (RR) e intervalos de confianza (IC) de $95 \%$, según diversas circunstancias maternas y gestacionales

\begin{tabular}{|c|c|c|c|c|c|}
\hline \multirow[b]{2}{*}{ Factor de riesgo } & \multirow{2}{*}{$\begin{array}{c}\text { Expuestos } \\
\text { en la muestra } \\
(\%)\end{array}$} & \multicolumn{2}{|c|}{$\%$ con BPN } & \multirow[b]{2}{*}{$\mathrm{RR}^{\mathrm{a}}$} & \multirow[b]{2}{*}{ IC } \\
\hline & & Expuestos & $\begin{array}{c}\text { No } \\
\text { expuestos }\end{array}$ & & \\
\hline \multicolumn{5}{|l|}{ Edad gestacional } & 1,8 a 4,1 \\
\hline $\begin{array}{l}\text { Intervalo intergenésico } \\
\text { previo } \leq 12 \text { meses }\end{array}$ & 4,6 & 20,0 & 8,7 & 2,3 & 1,2 a 4,3 \\
\hline $\begin{array}{l}\text { Gestación previa con } \\
\text { resultado desfavorable }\end{array}$ & 12,2 & 14,9 & 8,2 & 1,8 & 1,1 a 2,9 \\
\hline Estatura materna $\leq 1,5 \mathrm{~m}$ & 10,3 & 17,0 & 9,5 & 1,8 & 0,9 a 3,6 \\
\hline $\begin{array}{l}\text { Hipertensión durante la } \\
\text { gestación }\end{array}$ & 7,0 & 14,5 & 8,7 & 1,7 & 0,9 a 3,1 \\
\hline $\begin{array}{l}\text { Tabaquismo durante la } \\
\text { gestación } \\
\text { Edad materna } \leq 20 \text { ó }\end{array}$ & 24,7 & 12,3 & 7,9 & 1,6 & 1,0 a 2,3 \\
\hline $\begin{array}{l}\geq 36 \text { años } \\
\text { Atención prenatal }\end{array}$ & 31,5 & 11,9 & 7,8 & 1,5 & 1,0 a 2,3 \\
\hline $\begin{array}{l}<6 \text { consultas } \\
\text { Más de tres embarazos }\end{array}$ & 52,1 & 10,1 & 8,0 & 1,3 & 0,8 a 1,9 \\
\hline previos & 17,2 & 8,2 & 9,3 & 0,9 & 0,5 a 1,5 \\
\hline
\end{tabular}

a Riesgo relativo calculado para cada factor de riesgo como razón de frecuencias expuestos/no expuestos.

CUADRO 2. Estimación puntual e intervalo de confianza (IC) de $95 \%$ de la razón de posibilidades (odds ratio, $\psi$ ) de bajo peso al nacer (BPN) en modelos de regresión logística con una sola variable explicativa, aplicados a una muestra de 1023 neonatos dados a luz en Salvador, Bahia, 1987-1988

\begin{tabular}{lccc}
\hline \multicolumn{1}{c}{ Factor de riesgo } & $\psi$ & $\begin{array}{c}\text { Valor } \\
P\end{array}$ & $\begin{array}{c}\text { IC95\% } \\
\text { para } \psi\end{array}$ \\
\hline Edad gestacional $<38$ semanas & 3,0 & 0,00 & 1,9 a 4,8 \\
Intervalo intergenésico previo $\leq 12$ meses & 2,6 & 0,01 & 1,2 a 5,7 \\
Resultado desfavorable en gestación previa & 2,0 & 0,02 & 1,1 a 3,4 \\
Estatura materna $\leq 1,5 \mathrm{~m}$ & 2,0 & 0,12 & 0,8 a 4,5 \\
Hipertensión durante la gestación & 1,8 & 0,11 & 0,9 a 3,6 \\
Tabaquismo durante la gestación & 1,6 & 0,04 & 1,0 a 2,6 \\
Edad materna $\leq 20$ ó $\geq 36$ años & 1,6 & 0,04 & 1,0 a 2,5 \\
Atención prenatal $<6$ consultas & 1,3 & 0,26 & 0,8 a 2,0 \\
\hline
\end{tabular}

CUADRO 3. Razón de posibilidades (odds ratio, $\psi$ ) ajustada de bajo peso al nacer (BPN) para varios factores de riesgo, estimada mediante un modelo multifactorial de regresión logística (modelo final: $\chi^{2}=44,6$, con 6 g.l.), y riesgo atribuible poblacional (RAP) correspondiente (más detalles en el texto). Estimaciones puntuales e intervalos de confianza (IC) del $95 \%$ basados en una muestra de 1023 nacidos vivos en Salvador, Bahia, 1987-1988

\begin{tabular}{lcccc}
\hline \multicolumn{1}{c}{ Factor de riesgo } & $\psi$ & $\begin{array}{c}\text { Valor } \\
P\end{array}$ & IC95\% para $\psi$ & RAP (\%) \\
\hline $\begin{array}{l}\text { Edad gestacional }<38 \text { semanas } \\
\text { Intervalo intergenésico previo }\end{array}$ & 3,0 & $<0,01$ & 1,8 a 4,8 & 44 \\
$\quad \leq 12$ meses & 2,6 & 0,02 & 1,1 a 6,1 & 7 \\
$\begin{array}{l}\text { Resultado desfavorable } \\
\text { en el embarazo previo }\end{array}$ & 2,1 & 0,01 & 1,2 a 3,7 & 12 \\
Hipertensión durante el embarazo & 2,5 & 0,02 & 1,2 a 5,2 & 9 \\
Tabaquismo durante la gestación & 1,8 & 0,02 & 1,1 a 2,9 & 16 \\
Edad materna $\leq 20$ ó $\geq 36$ años & 1,9 & 0,01 & 1,1 a 3,0 & 21 \\
\hline
\end{tabular}

comparación con la "población" total de recién nacidos en Salvador durante un período dado. Sin embargo, la selección de la muestra por métodos no aleatorios puede hacer que estos neonatos no sean representativos de la población general en lo que respecta a estas características.

La edad gestacional de menos de 38 semanas fue el factor con mayor valor de $\psi$ ajustado y, siendo también el factor más frecuente en la muestra $(40 \%)$, es el de mayor RAP. El tabaquismo durante la gestación muestra también un RAP elevado por la alta proporción de fumadoras (25\%) en la muestra. El intervalo intergenésico corto presentó un valor elevado de $\psi$ en el modelo multifactorial, pero por la baja proporción de gestantes en las que se daba este factor de riesgo el RAP fue el mínimo (cuadro 3).

\section{DISCUSIÓN}

Dada la posibilidad de un fenómeno de confusión entre los factores de exposición mencionados, tratados dicotómicamente (sí/no) respecto a la presencia de BPN, en el presente estudio se utilizó una regresión logística para construir un modelo multifactorial en el que se controlaron estos factores. En el análisis multifactorial no se incluyeron variables socioeconómicas debido a que el efecto de la desigualdad social respecto al BPN es mediado en gran parte por las otras variables estudiadas. En otras investigaciones en las que se analizó la determinación social del BPN se observaron asociaciones con el ingreso familiar y la escolaridad materna. Cuando estas variables (entendidas como indicadores socioeconómicos) se ajustaron según otros factores de riesgo (atención prenatal, tabaquismo, número de partos previos, nutrición materna y edad gestacional), la asociación con el BPN dejó de ser estadísticamente significativa $(16,17,26)$. Según Barros et al. (26) esto se debe a que "la asociación clase social-BPN está casi totalmente mediada por otras variables".

El análisis de los datos mostró que uno de los factores de riesgo —más de 
tres gestaciones previas- no presentó asociación con el BPN y otros dos factores -estatura materna $\leq 1,5 \mathrm{~m}$ y número de consultas $<6$ en el período gestacional- no mostraron resultados estadísticamente significativos. Los demás factores estudiados — prematuridad, intervalo intergenésico corto, resultado desfavorable en la última gestación, hipertensión o tabaquismo durante el embarazo y madre demasiado joven o de edad avanzada- elevaron la incidencia de BPN cuando estuvieron presentes y se mantuvieron en el modelo final de regresión logística, con resultados estadísticamente significativos.

Cabe señalar que las estimaciones de $\psi$ ajustada en el método multifactorial corresponden a valores encontrados a partir de las demás variables presentes en el modelo. Así el valor ajustado de $\psi$ correspondiente al tabaquismo gestacional indica que independientemente de la exposición a los otros cinco factores presentes en el modelo, el hábito de fumar durante la gestación aumenta la incidencia de BPN casi al doble (1,8 veces).
Los factores de riesgo que fueron importantes en la determinación del BPN en su mayor parte han sido también hallados en otros estudios, aunque generalmente se han analizado de forma unifactorial, aisladamente en relación con el efecto investigado. Es destacable que un resultado desfavorable en la anterior gestación ha de considerarse como un marcador de riesgo de gestantes que probablemente presentan características adversas para la evolución normal de su gestación, que se pueden perpetuar en gestaciones posteriores. El intervalo intergenésico y la edad materna están directamente relacionados con las condiciones sociales y el acceso a la información y los servicios que permiten una elección más adecuada del momento más favorable para la gestación. La atención prenatal no puede modificar la exposición a estos tres factores de riesgo, pero puede ser importante para evitar o reducir la exposición al tabaco y para controlar la hipertensión, la evolución del embarazo y diversos factores potencialmente contribuyen- tes a la reducción de la duración gestacional.

El tabaquismo durante la gestación resultó también factor de riesgo en los modelos multifactoriales de determinación del BPN de Barros et al. (26) y Benício et al. (17). La edad gestacional ya había resultado un factor de riesgo importante en el análisis multifactorial realizado por Barros et al. (26). La edad materna no se había considerado en el estudio de Benício et al. (17).

Los factores de riesgo que presentan asociación con el BPN deben recibir especial atención en los programas que tienen como objetivo la disminución de la incidencia de BPN. Los factores que han de recibir mayor atención son aquellos en los que mayor es el RAP. El control de estos factores, es decir la reducción o la eliminación de la exposición, implicaría una intensa reducción del problema de salud pública. Junto con la situación socioeconómica, esos factores pueden utilizarse para detectar los embarazos de alto riesgo de BPN que, por tanto, requieren mayor atención de salud durante el período gestacional.

\section{REFERENCIAS}

1. Mata LJ, Urrutia JJ, Kronmal RA, Joplin C. Survival and psychical growth in infancy and early chilhood: study of birth weight and gestational age in a Guatemala Indian village. Am J Dis Child 1975;129:561-566.

2. Lasky RE, Lechtig A, Delgado H, Klein RE, Engle P, Yarbrough C, Martorell R. Birth weight and psychomotor performance in rural Guatemala. Am J Dis Child 1975;129:566-569.

3. Chandra R. Fetal malnutrition and postnatal immunocompetence. Am J Dis Child 1975;129: 450-454.

4. McCormick NC. The contribution of low birth weight to infant mortality and childhood morbidity. N Engl J Med 1985; 312:82-90.

5. Puffer RR, Serrano CV. Características de la mortalidad en la niñez. Washington, DC: Organización Panamericana de la Salud; 1973. (Publicación Científica 262).

6. Schwarcz R, Gonzalo Díaz A, Fescina R, Díaz Rossello JL, Belitzky R, Martell M. Bajo peso al nacer y mortalidad perinatal en maternidades de América Latina. En: Organización Panamericana de la Salud. Salud maternoinfantil y atención primaria en las Américas: hechos y tendencias. Washington, DC; 1984:105-117. (Publicación Científica 461)
7. Laurenti R, Buchala CM. Estudo de morbidade e mortalidade perinatal em maternidades: II, Mortalidade perinatal segundo peso ao nascer, idade materna, assistência prénatal e hábito de fumar da mae. Rev Saude Publica 1985;19:225-232.

8. Barros FC, Victora CG, Vaughan JP, Capellari MM. El riesgo perinatal en las ciudades del Tercer Mundo. Foro Mund Salud 1985;6: 369-372.

9. Lopes AAS, Port FK. The low birth weight hypothesis as a plausible explanation for the black-white differences in hypertension, noninsulin-dependent diabetes and end-stage renal disease. Am J Kidney Dis 1995;25:350-356.

10. Puffer RR, Serrano CV. Patterns of birthweight. Washington, DC: Pan American Health Organization; 1987. (Publicación Científica 504).

11. The incidence of low birth weight: an update. Wkly Epidemiol Rec 1984;59:205-211.

12. Loffredo LCM, Simôes MJS. Peso ao nascer e padrões de atendimento ao parto em município do Estado de São Paulo, Brasil, 1986. Rev Saude Publica 1990;24(1):80-83.

13. Victora CG, Grassi PR, Schmidt AM. Situação de saúde da criança em área da região sul do Brasil, 1980-1992: tendências temporais e distribuição espacial. Rev Saude Publica 1994; 28(6):423-432.

14. Mello Jorge MHP, Gotlieb SLD, Sioboll MLMS, Almeida MF, Latorre MRDO. Avaliação do sistema de informação sobre nascidos vivos e o uso de seus dados em epidemiologia e estatísticas de saúde. Rev Saude Publica 1993; 27(supl):2-24.

15. Monteiro MFG, Cervini R, comp. Perfil estatístico de crianças e maes no Brasil: aspectos de saúde e nutrição de crianças no Brasil, 1989. Rio de Janeiro: Instituto Brasileiro de Geografia e Estatística (IBGE); 1992.

16. Benício MH. Fatores de risco de baixo peso ao nascer em récem-nascidos vivos [tesis de doctorado]. Facultad de Medicina de la Universidad de São Paulo, Brasil, 1978.

17. Benício MH, Monteiro C, Souza J, Castilho E. Análise multivariada de fatores de risco para o baixo peso ao nascer em nascidos-vivos do município de São Paulo. Rev Saude Publica 1985;19:311-320.

18. Victora CG, Barros FC, Vaughan JP. Baixo peso ao nascer. En: Victora CG, Barros FE, Vaughan JP. Epidemiologia da desigualdade: um estudo longitudinal de 6000 crianças brasileiras. 2a ed. São Paulo: HUCITEC; 1989:44-58. 
19. Sinclair J, Saigal S. Nutritional influences in industrial societies. Am J Dis Child 1975; 129 (5):549-553.

20. Lechtig A, Delgado H, Lasky R, Klein RE, Engle PL, Yarbrough C, et al. Maternal nutrition and fetal growth in developing societies: socioeconomic factors. Am J Dis Child 1975; 129:434-437.

21. Lechtig A, Delgado H, Lasky R, Yarbrough C, Klein RE, Habicht J-P, et al. Maternal nutrition and fetal growth in developing countries. Am J Dis Child 1975;129:553-556.

22. Sinisterra Rodriguez OTS, Szarfarc SC, Benício $\mathrm{MH}$. Anemia e desnutrição maternas e sua relação com o peso ao nascer. Rev Saude Publica 1991;25:193-197.

23. Beisel WR. Synergistic effects of maternal malnutrition and infection on the infant. Am J Dis Child 1975;129:571-574.

24. Sever JL, Fuccillo DA, Ellenberg J, Gilkeson MR. Infection and low birth weight in an industrialized society. Am J Dis Child 1975; 129:557-558.

25. Urrutia JJ, Mata LJ, Trent F, Cruz JR, Villatoro E, Alexander RE. Infection and low birth weight in a developing country. Am J Dis Child 1975;129:558-561.

26. Barros FC, Victora CG, Vaughan JP, Estanislau HJ. Bajo peso al nacer en el municipio de Pelotas, Brasil: factores de riesgo. Bol Oficina Sanit Panam 1987;102(6):541-554.
27. Belizán JM, Nardín JC, Carroli G, Campodónico $\mathrm{L}$. Factores de riesgo de bajo peso al nacer en un grupo de embarazadas de Rosario, Argentina. Bol Oficina Sanit Panam 1989; 106(5):380-388.

28. Michileutte $\mathrm{R}$, et al. A comparison of risk assessment models for term and preterm low birth weight. Prev Med 1992;21:98-109.

29. Guimarães CM, De Siqueira AAF. Influência do número de gestações, hábito de fumar e intervalo interpartal sobre o peso ao nascer. Estud Goiania (Goiânia)1984;11:101-111.

30. Siqueira AAF, Santos JLF, Saqueto CG, Luz ET, Araújo MCA. Estado nutricional e hábito de fumar maternos, crescimento intra-uterino e pós-natal. Rev Saude Publica 1985;19:37-50.

31. Siqueira AAF, Santos JLF, Silva JF. Relação entre estado nutricional da gestante, fumo durante a gravidez, crescimento fetal e no primeiro ano de vida. Rev Saude Publica 1986; 20:421-434.

32. Aleixo Neto A. Efeitos do fumo na gravidez. Rev Saude Publica 1990;24:420-424.

33. Lee K, Ferguson RH, Corpuz MC, Gartner LM. Maternal age and incidence of low birth weight at term: a population study. Am J Obstetr Gynecol 1988;158:84-89.

34. Stüsser R, Paz G, Ortega M, Pineda S, Infante $\mathrm{O}$, Martin P, Ordóñez C. Riesgo de bajo peso al nacer en el Área Plaza de la Habana. Bol Oficina Sanit Panam 1993;114:229-241.
35. Brasil, Secretaria de Planejamento da Presidência da República. Fundação Instituto de Geografia e Estatística. Vol 5, tomo 12, Pesquisa Nacional por Amostra de Domicílios 1981: Regiões Metropolitanas. Rio de Janeiro: Instituto Brasileiro de Geografia e Estatística (IBGE); 1983.

36. The incidence of low birth weight: a critical review of available information. World Health Stat Q 1980;33:197-225.

37. Organização Mundial da Saúde. Manual de classificação estatística internacional de doenças, lesões e causas de óbitos; 9a rev 1975. São Paulo: Centro da OMS para Classificação de Doenças em Português; 1979.

38. Kleinbaum DG. Logistic regression: a self-learning text. New York: Springer Verlag; 1994

39. Rosner B. Fundamentals of biostatistics. Harvard University/Duxbury Press; 1995.

40. Rouquayrol MZ. Epidemiologia e saúde. Rio de Janeiro: MEDSI; 1993.

41. Lilienfeld AM, Lilienfeld DE. Foundations of epidemiology. New York: Oxford University Press; 1980.

Manuscrito recibido el 31 de enero de 1996 y aceptado para publicación en versión revisada el 19 de noviembre de 1996
ABSTRACT

Multifactorial analysis of the risk factors for low birthweight in Salvador, Bahia
This study is a multifactorial analysis of the risk factors for low birthweight in a group of newborns in an urban area of Brazil. A total of 1023 infants born in four maternity units in Salvador, Bahia, between July 1987 and February 1988 were included in the study. The sources of information were clinical histories and interviews with the mothers in the maternity units. The analysis was by means of logistic regression. In the final model the risk factors were the following: maternal age less than 21 years or more than 35; gestational age less than 38 weeks; unfavorable outcome of an earlier pregnancy; interval of 12 months or less since prior birth; tobacco smoking; and hypertension. The population attributable risk values for the risk factors included in the final model are presented. These factors should be used to identify pregnant women at high risk of giving birth to a low-birthweight baby, in order to provide them with more prenatal care. 